\title{
Chiral dynamics of the two $\Lambda(1405)$ states
}

\author{
D. Jido ${ }^{a, c}$, J.A. Oller ${ }^{b}$, E. Oset ${ }^{c}$, A. Ramos ${ }^{d}$ and U.-G. Meißner ${ }^{e}$ \\ ${ }^{a}$ Research Center for Nuclear Physics (RCNP), Osaka University, \\ Ibaraki, Osaka 567-0047, Japan \\ ${ }^{b}$ Departamento de Física, Universidad de Murcia, 30071 Murcia, Spain \\ ${ }^{c}$ Departamento de Física Teórica and IFIC, Centro Mixto Universidad de Valencia-CSIC, \\ Institutos de Investigación de Paterna, Aptd. 22085, 46071 Valencia, Spain \\ ${ }^{d}$ Departament d'Estructura i Constituents de la Matèria, Universitat de Barcelona, \\ Diagonal 647, 08028 Barcelona, Spain \\ ${ }^{e}$ Universität Bonn, Helmholtz-Institut für Strahlen- und Kernphysik (Theorie) \\ Nußalle 14-16, D-53115 Bonn, Germany
}

February 4, 2008

\begin{abstract}
Using a chiral unitary approach for the meson-baryon interactions, we show that two octets of $J^{\pi}=1 / 2^{-}$baryon states, which are degenerate in the limit of exact $\mathrm{SU}(3)$ symmetry, and a singlet are generated dynamically. The SU(3) breaking produces the splitting of the two octets, resulting in the case of strangeness $S=-1$ in two poles of the scattering matrix close to the nominal $\Lambda(1405)$ resonance. These poles are combinations of the singlet state and the octets. We show how actual experiments see just one effective resonance shape, but with properties which change from one reaction to another.
\end{abstract}




\section{Introduction}

The $\Lambda(1405)$ resonance has been a long-standing example of a dynamically generated resonance appearing naturally in scattering theory with coupled meson-baryon channels with strangeness $S=-1$ [1]. Modern chiral formulations of the meson-baryon interaction within unitary frameworks all lead to the generation of this resonance, which is seen as a near Breit-Wigner form in the mass distribution of $\pi \Sigma$ states with isospin $I=0$ in hadronic production processes [2, 5, 3, 4]. Yet, it was shown that in some models one could obtain two poles close to the nominal $\Lambda(1405)$ resonance, as it was the case within the cloudy bag model in Ref. [6]. Also, in the investigation of the poles of the scattering matrix in Ref. [3], within the context of chiral dynamics, it was found that there were two poles close to the nominal $\Lambda(1405)$ resonance both contributing to the $\pi \Sigma$ invariant mass distribution. This was also the case in Refs. [7,8], where two poles are obtained with similar properties as to their masses, widths and partial decay widths compared to those of the previous works.

The purpose of this paper is to investigate further the origin of these two poles, their nature and why there seems to be only one resonance in actual experiments. We furthermore suggest new experiments which could reveal the presence of these two states.

The manuscript is organized as follows. In Section 2, we briefly summarize the salient features of the chiral unitary coupled channel approach for the interactions between the octet of Goldstone bosons and the octet of the lowest baryons. The poles in the corresponding meson-baryon scattering matrix are discussed in Section 3, with particular emphasis on the group structure of the dynamically generated resonances in the $\mathrm{SU}(3)$ limit. The relation between these states and their couplings in that limit and in the physical case is further elaborated on in Section 4. In Section 5, we show how these poles manifest themselves in physical observables, and how different experiments are able to unravel the two poles generating the $\Lambda(1405)$. Some conclusions and an outlook are given in Section 6 . Some technicalities are relegated to the appendices.

\section{Description of the meson baryon interactions}

Starting from the chiral Lagrangians for meson-baryon interactions [9] and using the N/D method to obtain a scattering matrix fulfilling exactly unitarity in coupled channels [3, the full set of transition matrix elements with the coupled channels in $S=-1, K^{-} p, \bar{K}^{0} n$, $\pi^{0} \Lambda, \pi^{0} \Sigma^{0}, \pi^{+} \Sigma^{-}, \pi^{-} \Sigma^{+}, \eta \Lambda, \eta \Sigma^{0}, K^{0} \Xi^{0}$ and $K^{+} \Xi^{-}$, is given in matrix form by

$$
T=[1-V G]^{-1} V
$$

Here, the matrix $V$, obtained from the lowest order meson-baryon chiral Lagrangian, contains the Weinberg-Tomozawa or seagull contribution, as employed e.g. in Ref. [10],

$$
V_{i j}=-C_{i j} \frac{1}{4 f^{2}}\left(2 \sqrt{s}-M_{i}-M_{j}\right)\left(\frac{M_{i}+E}{2 M_{i}}\right)^{1 / 2}\left(\frac{M_{j}+E^{\prime}}{2 M_{j}}\right)^{1 / 2},
$$


where the $C_{i j}$ coefficients are given in Ref. [5], and an averaged meson decay constant $f=1.123 f_{\pi}$ is used [10], with $f_{\pi}=92.4 \mathrm{MeV}$ the weak pion decay constant. At lowest order in the chiral expansion all the baryon masses are equal to the one in the chiral limit, $M_{0}$, nevertheless in Ref. [10] the physical baryon masses, $M_{i}$, were used and these are the ones appearing in Eq. (2). In addition to the Weinberg-Tomozawa term, one also has at the same order in the chiral expansion the direct and exchange diagrams considered in Ref. 3. These are suppressed at low energies by powers of the three-momenta and meson masses over $M_{i}$, the leading one being just linear. However, their importance increases with energy and around $\sqrt{s} \simeq 1.5 \mathrm{GeV}$ they can be as large as a $20 \%$ of the seagull term.

The diagonal matrix $G$ stands for the loop function of a meson and a baryon and is defined by a dispersion relation in terms of phase space with a cut starting at the corresponding threshold $s_{l}$, namely [3]:

$$
\begin{aligned}
G(s)_{l} & =G\left(s_{0}\right)_{l}-\frac{s-s_{0}}{\pi} \int_{s_{l}}^{\infty} d s^{\prime} \frac{\rho\left(s^{\prime}\right)_{l}}{\left(s^{\prime}-s-i 0^{+}\right)\left(s^{\prime}-s_{0}\right)}, \\
\rho(s)_{l} & =\frac{M_{i} q_{l}}{4 \pi \sqrt{s}}
\end{aligned}
$$

and $G\left(s_{0}\right)_{l}$ is a subtraction constant. The above expression corresponds to the loop function of a meson and a baryon once the logarithmic divergent constant is removed:

$$
G_{l}=i \int \frac{d^{4} q}{(2 \pi)^{4}} \frac{M_{l}}{E_{l}(\vec{q})} \frac{1}{k^{0}+p^{0}-q^{0}-E_{l}(\vec{q})+i \epsilon} \frac{1}{q^{2}-m_{l}^{2}+i \epsilon} .
$$

The analytical properties of $G$ are properly kept when evaluating the previous loop function in dimensional regularization. Using dimensional regularization and removing the divergent constant piece leads to

$$
\begin{aligned}
G_{l}=i 2 M_{l} \int \frac{d^{4} q}{(2 \pi)^{4}} \frac{1}{(P-q)^{2}-M_{l}^{2}+i \epsilon} \frac{1}{q^{2}-m_{l}^{2}+i \epsilon} \\
=\frac{2 M_{l}}{16 \pi^{2}}\left\{a_{l}(\mu)+\ln \frac{M_{l}^{2}}{\mu^{2}}+\frac{m_{l}^{2}-M_{l}^{2}+s}{2 s} \ln \frac{m_{l}^{2}}{M_{l}^{2}}+\right. \\
+\frac{q_{l}}{\sqrt{s}}\left[\ln \left(s-\left(M_{l}^{2}-m_{l}^{2}\right)+2 q_{l} \sqrt{s}\right)+\ln \left(s+\left(M_{l}^{2}-m_{l}^{2}\right)+2 q_{l} \sqrt{s}\right)\right. \\
\left.\left.\quad-\ln \left(-s+\left(M_{l}^{2}-m_{l}^{2}\right)+2 q_{l} \sqrt{s}\right)-\ln \left(-s-\left(M_{l}^{2}-m_{l}^{2}\right)+2 q_{l} \sqrt{s}\right)\right]\right\},(6
\end{aligned}
$$

where $\mu$ is the scale of dimensional regularization. For a given value of this scale, the subtraction constant $a_{i}(\mu)$ is determined so that the results are finally scale independent.

The loop function represented by Eq. (5) was calculated in Ref. [5] with a cut-off regularization, similarly as previously done in meson-meson scattering [11]. The values of the $a_{i}$ constants in Eq. (6) are found to be around -2 to agree with the results of the cut-off method for cut-off values of the order of the mass of the $\rho(770)$ [3], which we call 
of natural size. Indeed, in Ref. [10] it was found that with the values for the subtraction constants

$$
\begin{array}{lll}
a_{\bar{K} N}=-1.84 & a_{\pi \Sigma}=-2.00 & a_{\pi \Lambda}=-1.83 \\
a_{\eta \Lambda}=-2.25 & a_{\eta \Sigma}=-2.38 & a_{K \Xi}=-2.67 .
\end{array} .
$$

one reproduces the results for the $G$ functions obtained in Ref. [5] with a cut-off of 630 $\mathrm{MeV}$.

It is further interesting to consider the large $N_{c}$ counting of the different terms present in Eq.(11). From Eq. (21), since $f^{2} \propto N_{c}$ and also $M_{B_{i}} \propto N_{c}$, it follows that $V_{i j}$ is $\mathcal{O}\left(N_{c}^{-1}\right)$ in the large $N_{c}$ counting. Here, one has to expand Eq. (22) in the limit of $M_{i} \rightarrow \infty$, taking into account that $\sqrt{s}=\sqrt{q^{2}+M_{i}^{2}}+\sqrt{q^{2}+m_{i}^{2}}$, so that the baryon masses disappear when subtracted from $2 \sqrt{s}$ in the first term between brackets of this equation which is then $\mathcal{O}\left(N_{c}^{0}\right)$. Since the other terms between brackets are also $\mathcal{O}\left(N_{c}^{0}\right)$ it follows that $V_{i j}$ is $\mathcal{O}\left(N_{c}^{-1}\right)$ because of the factor of $f^{2}$ appearing in the denominator. In the same way, by expanding Eq. (6) in the limit $M_{l} \rightarrow \infty$, a term linear in $M_{l}$ appears so that $G_{l}$ seems to be $\mathcal{O}\left(N_{c}\right)$. This term is energy-independent and is given by the combination:

$$
\frac{M_{l}}{8 \pi^{2}}\left(a_{l}(\mu)+\ln \frac{M_{l}^{2}}{\mu^{2}}\right) .
$$

The energy-dependent terms are $\mathcal{O}\left(N_{c}^{0}\right)$. In ref. [3] it was shown that

$$
a_{l}(\mu)=-2 \ln \left(1+\sqrt{1+\frac{M_{l}^{2}}{\mu^{2}}}\right)+\mathcal{O}\left(\frac{1}{M_{l}}\right),
$$

where the scale $\mu$ can be chosen such that it corresponds to a hypothetical three-momentum cut-off with a natural value around $M_{\rho}$ used to evaluate $G_{l}(s)$, as e.g. in ref. [5]. Taking the limit $M_{l} \rightarrow \infty$ in this equation one then has:

$$
a_{l}(\mu) \rightarrow-\ln \frac{M_{l}^{2}}{\mu^{2}},
$$

and hence the leading combination of Eq.(8) reduces to an $\mathcal{O}\left(N_{c}^{0}\right)$ contribution as the rest of the terms so that in this case $G_{l}$ is finally $\mathcal{O}\left(N_{c}^{0}\right)$. This is also the order one would infer naturally from Eq.(4) by simply applying the scaling properties $M_{l} \backsim \mathcal{O}\left(N_{c}\right)$ and $q \backsim \mathcal{O}\left(N_{c}^{0}\right)$ to the integral and then taking the accompanying subtraction constant $G_{l}\left(s_{0}\right)$ of the same order as that of the integral. Since from the studies of refs. [5, 3, 10, it is clear that one can consider the subtractions constant $a_{l}$ as originating from a cut-off as in Eq. (9), then we infer that the $G_{l}(s)$ function must be counted as $\mathcal{O}\left(N_{c}^{0}\right)$. The important point for us is that then the product $V G$, appearing in Eq. (II), is $\mathcal{O}\left(N_{c}^{-1}\right)$ and then suppressed in the large $N_{c}$ limit with respect to the identity. This situation is similar to that of the meson-meson case, where $V G$ is as well $\mathcal{O}\left(N_{c}^{-1}\right)$ [13. Thus, the dynamically generated resonances disappear in the limit of large number of colours. Were the subtraction constants not generated through a relation like that of Eq.(9), which implies a value of $a_{l}$ around 
-2 , then $V G$ would be $\mathcal{O}\left(N_{c}^{0}\right)$ and would not be suppressed as compared to the identity so that the poles would survive the large $N_{c}$ limit. Thus, as a result of this discussion, we want to emphasize that the set of resonances with $S=-1$ generated dynamically within our approach and to be presented in detail below, are suppressed in the large $N_{c}$ limit and are not suited to a large $N_{c}$ expansion as that employed recently in ref. [12.

\section{Poles of the T-matrix}

The study of Ref. 10] showed the presence of poles in Eq. (11) around the $\Lambda(1405)$ and the $\Lambda(1670)$ for isospin $I=0$ and around the $\Sigma(1620)$ in $I=1$. The same approach in $S=-2$ leads to the resonance $\Xi(1620)$ [14] and in $S=0$ to the $N^{*}(1535)$ [15], this latter one also generated dynamically in Ref. [16]. One is thus tempted to consider the appearance of a singlet and an octet of meson-baryon resonances. Nevertheless, the situation is more complicated because indeed in the $\mathrm{SU}(3)$ limit there are two octets and not just one, as we discuss below. As a matter of fact, the $\Lambda(1405)$ is a mixture of a singlet and an octet, and not just a singlet as assumed in Ref. 12. The presence of these multiplets was already discussed in Ref. [3] after obtaining poles with $S=-1$ in the $I=1$ channel, with mass around $1430 \mathrm{MeV}$, and two poles with $I=0$, of masses around that of the $\Lambda(1405)$. Similar ideas have been exploited in the meson-meson interaction where a nonet of dynamically generated mesons, made of the $\sigma(500), f_{0}(980), a_{0}(980)$ and $\kappa(900)$, has been obtained [11, 18, 13, 17.

The appearance of a multiplet of dynamically generated mesons and baryons seems most natural once a state of the multiplet appears. Indeed, one must recall that the chiral Lagrangians are obtained from the combination of the octet of pseudoscalar mesons (the pions and partners) and the octet of stable baryons (the nucleons and partners). The $\mathrm{SU}(3)$ decomposition of the combination of two octets tells us that

$$
8 \otimes 8=1 \oplus 8_{s} \oplus 8_{a} \oplus 10 \oplus \overline{10} \oplus 27 .
$$

Thus, on pure SU(3) grounds, should we have a SU(3) symmetric Lagrangian, one can expect e.g. one singlet and two octets of resonances, the symmetric and antisymmetric ones. Actually in the case of the meson-meson interactions only the symmetric octet appears in S-wave because of Bose statistics, but in the case of the meson-baryon interactions, where the building blocks come from two octets of different nature, both the symmetric and antisymmetric octets could appear and there is no reason why they should be degenerate in principle.

The lowest order of the meson-baryon chiral Lagrangian is exactly SU(3) invariant if all the masses of the mesons, or equivalently the quark masses, are set equal. As stated above [see Eq. (2)], in Ref. [10] the baryon masses take their physical values, although strictly speaking at the leading order in the chiral expansion they should be equal to $M_{0}$. For Eq. (2) being SU(3) symmetric, all the baryons masses $M_{i}$ must be set equal as well. When all the meson and baryon masses are equal, and these common masses are employed in evaluating the $G_{l}$ functions, together with equal subtraction constants $a_{l}$, the $T$-matrix 
obtained from Eq. (11) is also $\mathrm{SU}(3)$ symmetric. In appendix A we show that in the $\mathrm{SU}(3)$ limit the subtraction constants $a_{l}$ are independent of the physical channel.

If we do such an SU(3) symmetry approximation and look for poles of the scattering matrix, we find poles corresponding to the octets and singlet. The surprising result is that the two octet poles are degenerate as a consequence of the dynamics contained in the chiral Lagrangians. Indeed, if we evaluate the matrix elements of the transition potential $V$ in a basis of $\mathrm{SU}(3)$ states,

$$
V_{\alpha \beta}=\sum_{i, j}\langle i, \alpha\rangle C_{i j}\langle j, \beta\rangle,
$$

where $\langle i, \alpha\rangle$ are the $\mathrm{SU}(3)$ Clebsch-Gordan coefficients and $C_{i j}$ the coefficients in Eq. (2), we obtain:

$$
V_{\alpha \beta}=\operatorname{diag}(6,3,3,0,0,-2),
$$

taking the following order for the irreducible representations: $1,8_{s}, 8_{a}, 10, \overline{10}$ and 27 .

Hence we observe that the states belonging to different irreducible representations do not mix, and the scattering amplitude of the two octets is the same at Born level (i.e. to leading order in the chiral expansion). Thus the two octets appear degenerate after the unitarized resummation. The coefficients in Eq. (13) clearly illustrate why there are no bound states in the $10, \overline{10}$ and 27 representations. Indeed, considering the minus sign in Eq. (21), a negative sign in Eq. (13) means repulsion. In practice, the same chiral Lagrangians allow for $\mathrm{SU}(3)$ breaking. In the case of Refs. [5, 10] the breaking appears because both in the $V_{i j}$ transition potentials as in the $G_{l}$ loop functions one uses the physical masses of the particles as well as different subtraction constants in $G_{l}$, corresponding to the use of a unique cut-off in all channels. In Ref. 3. the physical masses are also used in the $G_{l}$ functions, although these functions are evaluated with a unique subtraction constant as corresponds to the $\mathrm{SU}(3)$ limit, see appendix A. In addition the $V_{i j}$ transition potentials are evaluated strictly at lowest order in the chiral expansion so that a common baryon mass is used and the one baryon exchange diagrams, both direct and crossed, are included. In both approaches, physical masses are used to evaluate the $G_{l}$ loop functions so that unitarity is fulfilled exactly and the physical thresholds for all channels are respected. This is important in a multichannel problem like the present case. In general terms, additional SU(3) breaking contributions can be included systematically when evaluating the interacting kernel $V$ in a chiral expansion of the meson-baryon interactions, as explained in ref. [3]. The lowest order $\mathrm{SU}(3)$ breaking corrections occur when $V$ is evaluated at next-to-leading order from the $\mathcal{O}\left(p^{2}\right)$ meson-baryon Lagrangian, with some of these terms already included in Ref. [19. Other $\mathrm{SU}(3)$ breaking effects as e.g. those arising from the difference between the weak decay constants of the pseudoscalars appear at $\mathcal{O}\left(p^{3}\right)$ in $V$. The systematic inclusion of such higher order corrections is beyond the present study but should be considered in the future.

By following the approach of Ref. [10] and using the physical masses of the baryons and the mesons, the position of the poles change and the two octets split apart in four 


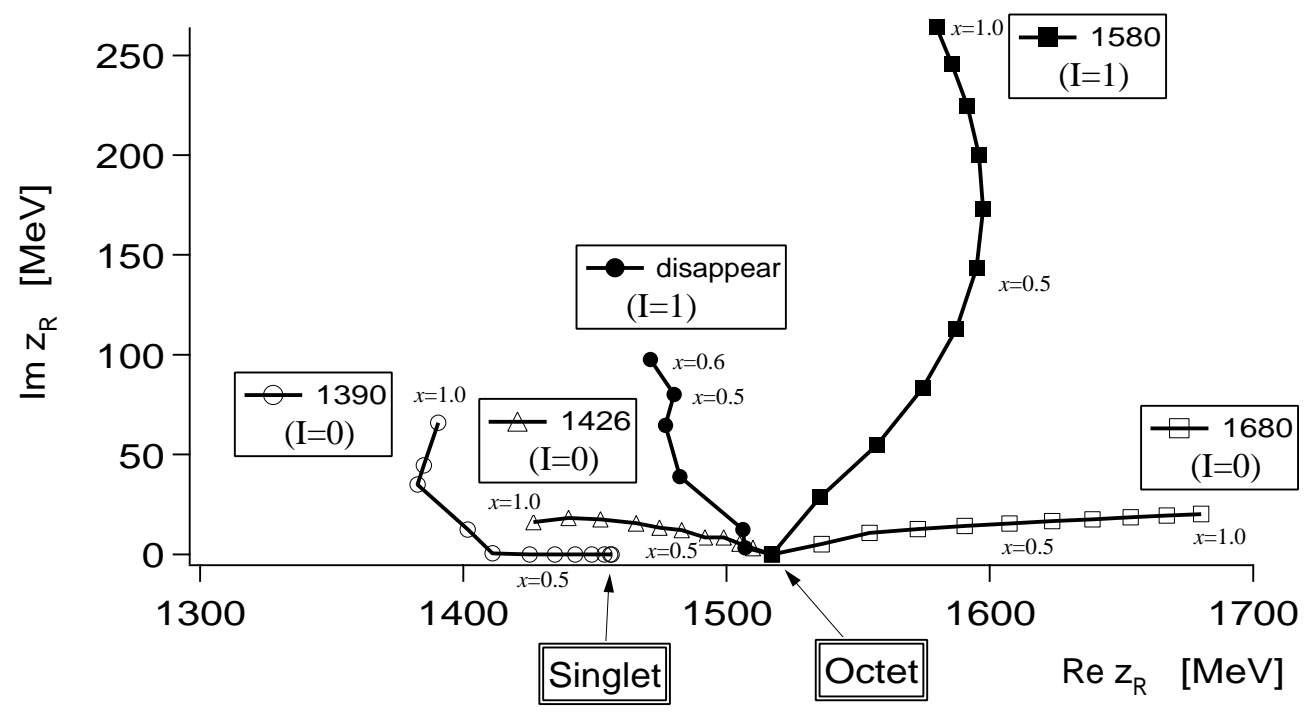

Figure 1: Trajectories of the poles in the scattering amplitudes obtained by changing the $\mathrm{SU}(3)$ breaking parameter $x$ gradually. At the $\mathrm{SU}(3)$ symmetric limit $(x=0)$, only two poles appear, one is for the singlet and the other for the octet. The symbols correspond to the step size $\delta x=0.1$.

branches, two for $I=0$ and two for $I=1$, as one can see in Fig. 11. In the figure we show the trajectories of the poles as a function of a parameter $x$ that breaks gradually the $\mathrm{SU}(3)$ symmetry up to the physical values. The dependence of masses and subtraction constants on the parameter $x$ is given by

$$
\begin{aligned}
M_{i}(x) & =M_{0}+x\left(M_{i}-M_{0}\right), \\
m_{i}^{2}(x) & =m_{0}^{2}+x\left(m_{i}^{2}-m_{0}^{2}\right), \\
a_{i}(x) & =a_{0}+x\left(a_{i}-a_{0}\right),
\end{aligned}
$$

where $0 \leq x \leq 1$. For the baryon masses, $M_{i}(x)$, the breaking of the $\mathrm{SU}(3)$ symmetry follows linearly, while for the meson masses, $m_{i}(x)$, the law is quadratic in the masses, since in the QCD Lagrangian the flavor SU(3) breaking appears in the quark mass terms and the squares of the meson masses depend on the quark masses linearly. In the calculation of Fig. 1. the values $M_{0}=1151 \mathrm{MeV}, m_{0}=368 \mathrm{MeV}$ and $a_{0}=-2.148$ are used.

The complex poles, $z_{R}$, appear in unphysical sheets. In the present search we follow the strategy of changing the sign of the momentum $q_{l}$ in the $G_{l}(z)$ loop function of Eq. (6) for the channels which are open at an energy equal to $\operatorname{Re}(z)$.

The splitting of the two $I=0$ octet states is very interesting. One moves to higher energies to merge with the $\Lambda(1670)$ resonance and the other one moves to lower energies to create a pole, quite well identified below the $\bar{K} N$ threshold, with a narrow width. In appendix B we show explicitly how for small values of the symmetry breaking parameter $x$ the poles move away from their values in the $\mathrm{SU}(3)$ symmetry limit. We should also note 
that when for some values of $x$ the trajectory crosses the $\bar{K} N$ threshold $(\sim 1435 \mathrm{MeV})$ the pole fades away but it emerges again clearly for values of $x$ close to 1 . On the other hand, the singlet also evolves to produce a pole at low energies with a quite large width.

We note that the singlet and the $I=0$ octet states appear nearby in energy and one of the purposes of this paper is, precisely, to point out the fact that what experiments actually see is a combination of the effect of these two resonances. This should be considered the main result of this paper.

Similarly as for the $I=0$ octet states, we can see that one branch of the $I=1$ states moves to higher energies while another moves to lower energies. The branch moving to higher energies finishes at what would correspond to the $\Sigma(1620)$ resonance when the physical masses are reached. The branch moving to lower energies fades away after a while when getting close to the $\bar{K} N$ threshold.

The model of Ref. [3] reproduces qualitatively the same results. However, this model also produces in the physical limit $(x=1)$ another $I=1$ pole having $\operatorname{Re}(z)=1401 \mathrm{MeV}$ if, in addition to changing the signs of the on-shell momenta in the $\pi \Lambda$ and $\pi \Sigma$ channels in accordance to the strategy mentioned above, the sign in the $\bar{K} N$ channel is also changed. This is legitimate in this case due to the proximity of the $\bar{K} N$ threshold to the position of the $I=1$ resonance of ref. [3] as compared to its width (see the later table 3). As we can see, the appearance of this pole is more sensitive to the details of the coupled channel approach and hence it is not so stable as the $I=0$ poles discussed above. Yet, the pole found in the model of Ref. 10] for values of $x$ below to 0.6, see Fig. 11 reflects, in the physical limit $x=1$, into a strong cusp structure of the $I=1$ amplitudes at the $\bar{K} N$ threshold, leading to a pronounced peak on top of a large background. In the model of Ref. [3], the presence of a pole in the Riemann sheet where the sign of the momenta in the three channels $(\pi \Lambda$, $\pi \Sigma, \bar{K} N)$ is changed enhances the structure of this peak, which shows the features of a resonant shape. Whether this enhancement in the $I=1$ amplitudes can be interpreted as a resonance or as a cusp, the fact that the strength of the $I=1$ amplitude around the $\Lambda(1405)$ region is not negligible should have consequences for reactions producing $\pi \Sigma$ pairs in that region. This has been illustrated for instance in Ref. 20], where the photoproduction of the $\Lambda(1405)$ via the reaction $\gamma p \rightarrow K^{+} \Lambda$ (1405) was studied. It was shown there that the different sign in the $I=1$ component of the $\left|\pi^{+} \Sigma^{-}\right\rangle,\left|\pi^{-} \Sigma^{+}\right\rangle$states leads, through interference between the $I=1$ and the dominant $I=0$ amplitudes, to different cross sections in the various charge channels, a fact that has been confirmed experimentally very recently $[21]$.

Once the pole positions are found, one can also determine the couplings of these resonances to the physical states by studying the amplitudes close to the pole and identifying them with

$$
T_{i j}=\frac{g_{i} g_{j}}{z-z_{R}} .
$$

The couplings $g_{i}$ are in general complex valued numbers. In Tables 1 and 2 we summarize the pole positions and the complex couplings $g_{i}$ obtained from the model of Ref. [10] for isospin $I=0$ and $I=1$, respectively.

We now consider the results obtained from the model of Ref. 3]. Making use of their 
Table 1: Pole positions and couplings to $I=0$ physical states from the model of Ref. 10]

\begin{tabular}{|c|cc|cc|cc|}
\hline$z_{R}$ & \multicolumn{2}{|c|}{$1390+66 i$} & \multicolumn{2}{|c|}{$1426+16 i$} & \multicolumn{2}{c|}{$1680+20 i$} \\
$(I=0)$ & $g_{i}$ & $\left|g_{i}\right|$ & $g_{i}$ & $\left|g_{i}\right|$ & $g_{i}$ & $\left|g_{i}\right|$ \\
\hline$\pi \Sigma$ & $-2.5-1.5 i$ & 2.9 & $0.42-1.4 i$ & 1.5 & $-0.003-0.27 i$ & 0.27 \\
$\bar{K} N$ & $1.2+1.7 i$ & 2.1 & $-2.5+0.94 i$ & 2.7 & $0.30+0.71 i$ & 0.77 \\
$\eta \Lambda$ & $0.010+0.77 i$ & 0.77 & $-1.4+0.21 i$ & 1.4 & $-1.1-0.12 i$ & 1.1 \\
$K \Xi$ & $-0.45-0.41 i$ & 0.61 & $0.11-0.33 i$ & 0.35 & $3.4+0.14 i$ & 3.5 \\
\hline
\end{tabular}

Table 2: Pole position and couplings to $I=1$ physical states from the model of Ref. [10]

\begin{tabular}{|c|cc|}
\hline$z_{R}$ & \multicolumn{2}{|c|}{$1579+264 i$} \\
$(I=1)$ & $g_{i}$ & $\left|g_{i}\right|$ \\
\hline$\pi \Lambda$ & $1.4+1.5 i$ & 2.0 \\
$\pi \Sigma$ & $-2.2-1.5 i$ & 2.7 \\
$\bar{K} N$ & $-1.1-1.1 i$ & 1.6 \\
$\eta \Sigma$ & $1.2+1.4 i$ & 1.9 \\
$K \Xi$ & $-2.5-2.4 i$ & 3.5 \\
\hline
\end{tabular}

set I of parameters, which correspond to a baryon mass $M_{0}=1286 \mathrm{MeV}$ and a meson decay constant $f=0.798 f_{\pi}=74.1 \mathrm{MeV}$, both in the chiral limit, together with a common subtraction constant $a=-2.23$, the results obtained for $I=0$ and $I=1$ are displayed in Tables 3 and 4 , respectively.

We can see that there is a qualitative agreement among both models, especially in the case of $I=0$. We observe that the second resonance with $I=0$ couples strongly to $\bar{K} N$ channel, while the first resonance couples more strongly to $\pi \Sigma$. The results for $I=0$ shown in Tables 11, 3] resemble much those obtained in Ref. 6] and Ref. [8] where two resonances are also found close to $1405 \mathrm{MeV}$, with the one at lower energies having a larger width than the second and a stronger coupling to $\pi \Sigma$, while the resonance at higher energies being narrower and coupling mostly to $\bar{K} N$.

\section{$4 \mathrm{SU}(3)$ considerations}

While in the discussion following Fig. 1 we have identified the states as octet and singlet because of their origin, it is clear that there is some mixing. In fact we can make a basis transformation and find the coupling of the resonances to the $\mathrm{SU}(3)$ meson-baryon states. We write the matrix

$$
\tilde{T}=U^{\dagger} T U
$$

where $U$ is the unitary matrix of the $\mathrm{SU}(3)$ Clebsch-Gordan coefficients $\langle i, \gamma\rangle$ with $i$ the indices for the physical states and $\gamma$ denoting a subset of the meson-baryon $\mathrm{SU}(3)$ states 
Table 3: Pole positions and couplings to $I=0$ physical states from the model of Ref. [3]

\begin{tabular}{|c|cc|cc|cc|}
\hline$z_{R}$ & \multicolumn{2}{|c|}{$1379+27 i$} & \multicolumn{2}{|c|}{$1434+11 i$} & \multicolumn{2}{|c|}{$1692+14 i$} \\
$(I=0)$ & $g_{i}$ & $\left|g_{i}\right|$ & $g_{i}$ & $\left|g_{i}\right|$ & $g_{i}$ & $\left|g_{i}\right|$ \\
\hline$\pi \Sigma$ & $-1.76-0.62 i$ & 1.87 & $-0.56-1.02 i$ & 1.16 & $-0.08-0.32 i$ & 0.33 \\
$\bar{K} N$ & $0.86+0.70 i$ & 1.11 & $-1.74+0.63 i$ & 1.85 & $0.32+0.41 i$ & 0.52 \\
$\eta \Lambda$ & $0.19+0.33 i$ & 0.38 & $-1.20+0.23 i$ & 1.23 & $-0.83-0.19 i$ & 0.85 \\
$K \Xi$ & $-0.52-0.19 i$ & 0.55 & $-0.20-0.30 i$ & 0.36 & $3.87+0.05 i$ & 3.87 \\
\hline
\end{tabular}

Table 4: Pole positions and couplings to $I=1$ physical states from the model of Ref. [3]

\begin{tabular}{|c|cc|cc|}
\hline$z_{R}$ & \multicolumn{2}{|c|}{$1401+40 i$} & \multicolumn{2}{|c|}{$1488+114 i$} \\
$(I=1)$ & $g_{i}$ & $\left|g_{i}\right|$ & $g_{i}$ & $\left|g_{i}\right|$ \\
\hline$\pi \Lambda$ & $0.60+0.47 i$ & 0.76 & $0.98+0.84 i$ & 1.3 \\
$\pi \Sigma$ & $1.27+0.71 i$ & 1.5 & $-1.32-1.00 i$ & 1.7 \\
$\bar{K} N$ & $-1.24-0.73 i$ & 1.4 & $-0.89-0.57 i$ & 1.1 \\
$\eta \Sigma$ & $0.56+0.41 i$ & 0.69 & $0.58+0.29 i$ & 0.65 \\
$K \Xi$ & $0.12+0.05 i$ & 0.13 & $-1.63-0.91 i$ & 1.9 \\
\hline
\end{tabular}

having hypercharge, isospin and isospin projection compatible with those of the physical ones. Taking the resonances and couplings found from the model of Ref. [10] (Table 1) we find the results shown in Table 5 .

Table 5: Couplings of the $I=0$ bound states to the meson-baryon $\mathrm{SU}(3)$ basis states, obtained with the model of Ref. [10]

\begin{tabular}{|c|cc|cc|cc|}
\hline$z_{R}$ & \multicolumn{2}{|c|}{$1390+66 i$} & \multicolumn{2}{c|}{$1426+16 i$} & \multicolumn{2}{c|}{$1680+20 i$} \\
& \multicolumn{2}{c|}{ (evolved singlet) } & (evolved octet $8_{s}$ ) & (evolved octet $\left.8_{a}\right)$ \\
& $g_{\gamma}$ & $\left|g_{\gamma}\right|$ & $g_{\gamma}$ & $\left|g_{\gamma}\right|$ & $g_{\gamma}$ & $\left|g_{\gamma}\right|$ \\
\hline 1 & $2.3+2.3 i$ & 3.3 & $-2.1+1.6 i$ & 2.6 & $-1.9+0.42 i$ & 2.0 \\
$8_{s}$ & $-1.4-0.14 i$ & 1.4 & $-1.1-0.62 i$ & 1.3 & $-1.5-0.066 i$ & 1.5 \\
$8_{a}$ & $0.53+0.94 i$ & 1.1 & $-1.7+0.43 i$ & 1.8 & $2.6+0.59 i$ & 2.7 \\
27 & $0.25-0.031 i$ & 0.25 & $0.18+0.092 i$ & 0.21 & $-0.36+0.28 i$ & 0.4 \\
\hline
\end{tabular}

We observe that the physical singlet couples mostly to the singlet SU(3) state. This means that this physical state has retained largely the singlet nature it had in the $\mathrm{SU}(3)$ symmetric situation. The same is true for the physical $I=0$ antisymmetric octet shown in the last column. However, the couplings of the physical symmetric octet reveal that, due to its proximity to the singlet state, it has become mostly a singlet with some admixture of the symmetric and antisymmetric octets.

Alternatively, one can try to determine the $\mathrm{SU}(3)$ pole content of a physical pole by 
decomposing the corresponding physical resonance, denoted by $|A\rangle$, in terms of the bound states found in the $\mathrm{SU}(3)$ symmetric situation, denoted by $\left|\mu^{\prime}\right\rangle$, as

$$
|A\rangle=\sum_{\mu} C_{\mu}^{(A)}\left|\mu^{\prime}\right\rangle
$$

with

$$
\sum_{\mu}\left|C_{\mu}^{(A)}\right|^{2} \simeq 1
$$

where the expansion has been limited to the $\mathrm{SU}(3)$ bound states which, in the present case, are found in the irreducible $\mathrm{SU}(3)$ representations $1,8_{a}$ or $8_{s}$. The small admixture of the 27 representation, as seen in Table 5, and a possible small contribution of the continuum states have been neglected in writing Eqs. (17) and (18). We note that the state $|A\rangle$ has well defined isospin ( $I=0$ or 1$)$, third component of isospin and hypercharge $(Y=0)$, the same as the $\left|\mu^{\prime}\right\rangle$ state in the sum on the right hand side of Eq. (17). For simplicity, all these quantum numbers are included in the symbols $A$ and $\mu$. If we further designate by $|\gamma M B\rangle$ the basis state belonging to the $\gamma \mathrm{SU}(3)$ irreducible representation and made up by a meson and a baryon, as for instance the singlet $|1 M B\rangle=\frac{1}{\sqrt{8}} \mid K^{-} p+\bar{K}^{0} n+\pi^{+} \Sigma^{-}+\pi^{0} \Sigma^{0}+$ $\left.\pi^{-} \Sigma^{+}+\eta \Lambda+K^{+} \Xi^{-}+K^{0} \Xi^{0}\right\rangle$, we can write for the coupling $|A\rangle \rightarrow|\gamma M B\rangle \equiv g(A \rightarrow \gamma)$

$$
g(A \rightarrow \gamma)=\sum_{\mu} C_{\mu}^{(A)} g\left(\mu^{\prime} \rightarrow \gamma\right)
$$

In the $\mathrm{SU}(3)$ limit $g\left(\mu^{\prime} \rightarrow \gamma\right)=\delta_{\mu \gamma} g\left(\gamma^{\prime} \rightarrow \gamma\right)$, hence, up to first order in the $\mathrm{SU}(3)$ breaking parameter, we have

$$
g(A \rightarrow \gamma)=C_{\gamma}^{(A)} g\left(\gamma^{\prime} \rightarrow \gamma\right)
$$

where the couplings $g(A \rightarrow \gamma)$ for the $I=0$ resonances are those given in Table 5 . Therefore, once the couplings $g\left(\gamma^{\prime} \rightarrow \gamma\right)$ are known, the equation above together with the normalization requirement of Eq. (18), is sufficient to determine the coefficients $C_{\gamma}^{(A)}$ except for a global phase.

In order to calculate the couplings $g\left(\gamma^{\prime} \rightarrow \gamma\right)$ we consider the $\mathrm{SU}(3)$ limit corresponding to the physical meson and baryon masses. In order to do that we take the Gell-MannOkubo mass relations for the lightest octet of pseudoscalars and baryons for calculating the common meson and baryon masses in the $\mathrm{SU}(3)$ limit, see e.g. Refs. [22, 23]. These result to be $M_{B}=1150 \mathrm{MeV}$ for the baryons and $m_{0}=413 \mathrm{MeV}$ for the mesons. The pole positions of the singlet and octet $\left(8_{s}\right.$ and $\left.8_{a}\right)$, together with the corresponding residua, obtained from the two models studied in this work are

\begin{tabular}{|l|c|c|}
\hline & Ref. [10] & Ref. [3] \\
\hline singlet pole & $(1486,0)$ & $(1447,0)$ \\
octet pole & $(1556,0)$ & $(1516,0)$ \\
$g\left(1^{\prime} \rightarrow 1\right)$ & $(-3.29,0)$ & $(-3.05,0)$ \\
$g\left(8^{\prime} \rightarrow 8\right)$ & $(-1.87,0)$ & $(-2.65,0)$ \\
\hline
\end{tabular}


Taking the $g(A \rightarrow \gamma)$ couplings from Table 5, one obtains the following coefficients

\begin{tabular}{|c|l|l|l|l|l|l|}
\hline Pole & $C_{1}$ & $C_{8_{a}} / C_{1}$ & $C_{8_{s}} / C_{1}$ & $\left|C_{1}\right|^{2}$ & $\left|C_{8_{a}}\right|^{2}$ & $\left|C_{8_{s}}\right|^{2}$ \\
\hline $1390+66 i$ & 0.73 & $(0.41,0.12)$ & $(-0.43,0.34)$ & 0.53 & 0.18 & 0.29 \\
\hline $1426+16 i$ & 0.56 & $(0.62,0.27)$ & $(0.20,0.45)$ & 0.31 & 0.45 & 0.24 \\
\hline $1680+20 i$ & 0.34 & $(-0.75,-0.35)$ & $(0.43,0.11)$ & 0.12 & 0.68 & 0.20 \\
\hline
\end{tabular}

which again confirms that the first pole is mostly a singlet, the third one mostly an antisymmetric octet and the second one has become an even mixture of the three $\mathrm{SU}(3)$ poles. Following the same steps but now for the $I=1$ pole found in the model of Ref. [10], one obtains the coefficients

$$
\begin{array}{|c|l|l|l|l|}
\hline \text { Pole } & C_{8_{a}} & C_{8_{s}} / C_{8_{a}} & \left|C_{8_{a}}\right|^{2} & \left|C_{8_{s}}\right|^{2} \\
\hline 1579+264 i & 0.55 & (0.83,0.14) & 0.30 & 0.70 \\
\hline
\end{array}
$$

which show that this pole has retained the $8_{s}$ nature it had in the $\mathrm{SU}(3)$ symmetric situation (see Eq. (B.7) in appendix B). Similarly, the results obtained from the model of Ref. [3] are

\begin{tabular}{|c|l|l|l|l|l|l|}
\hline Pole & $C_{1}$ & $C_{8_{a}} / C_{1}$ & $C_{8_{s}} / C_{1}$ & $\left|C_{1}\right|^{2}$ & $\left|C_{8_{a}}\right|^{2}$ & $\left|C_{8_{s}}\right|^{2}$ \\
\hline $1379+27 i$ & 0.96 & $(0.15,0.11)$ & $(0.15,-0.19)$ & 0.92 & 0.03 & 0.05 \\
\hline $1434+11 i$ & 0.49 & $(0.64,0.77)$ & $(0.71,1.28)$ & 0.24 & 0.24 & 0.52 \\
\hline $1692+14 i$ & 0.48 & $(1.58,0.37)$ & $(0.78,0.16)$ & 0.23 & 0.63 & 0.14 \\
\hline
\end{tabular}

for the $I=0$ poles and

\begin{tabular}{|c|l|l|l|l|}
\hline Pole & $C_{8_{a}}$ & $C_{8_{s}} / C_{8_{a}}$ & $\left|C_{8_{a}}\right|^{2}$ & $\left|C_{8_{s}}\right|^{2}$ \\
\hline $1401+40 i$ & 0.81 & $(0.72,0.07)$ & 0.66 & 0.34 \\
\hline $1488+114 i$ & 0.59 & $(1.37,-0.06)$ & 0.35 & 0.65 \\
\hline
\end{tabular}

for the $I=1$ ones. In this model, for which $\mathrm{SU}(3)$ breaking is less pronounced, the physical poles retain better the irreducible $\mathrm{SU}(3)$ nature they had in the $\mathrm{SU}(3)$ symmetric situation.

If the basis transformation of Eq. (17) was complete, the sum of the moduli square of the coefficients should also be one along a column, which corresponds to expanding the $\mathrm{SU}(3)$ eigenstates in the physical basis of resonances. Within $20 \%$ this is true for all the columns in the tables of Eqs. (22), (24) and (25), except for the one corresponding to the $\mathrm{SU}(3)$ singlet state in the model of Ref. [3], which is overestimated in a $40 \%$ [see Eq. (24)].

\section{Influence of the poles on the physical observables}

It is important to see what would happen in an actual experiment. In order to see this, we first make a qualitative and intuitive exercise and then compare to the numerical results obtained using the model of Ref. [10]. Consider now two resonances, called $R_{1}$ and $R_{2}$, corresponding to the physical singlet and symmetric octet states. Take the complex 
couplings of Table 1 and construct the following amplitudes

$$
\begin{aligned}
& g_{\bar{K} N}^{R_{1}} \frac{1}{W-M_{R_{1}}+i \Gamma_{R_{1}} / 2} g_{\pi \Sigma}^{R_{1}}+g_{\bar{K} N}^{R_{2}} \frac{1}{W-M_{R_{2}}+i \Gamma_{R_{2}} / 2} g_{\pi \Sigma}^{R_{2}}, \\
& g_{\pi \Sigma}^{R_{1}} \frac{1}{W-M_{R_{1}}+i \Gamma_{R_{1}} / 2} g_{\pi \Sigma}^{R_{1}}+g_{\pi \Sigma}^{R_{2}} \frac{1}{W-M_{R_{2}}+i \Gamma_{R_{2}} / 2} g_{\pi \Sigma}^{R_{2}} .
\end{aligned}
$$

This would be basically equivalent to the amplitudes $T_{\bar{K} N \rightarrow \pi \Sigma}$ and $T_{\pi \Sigma \rightarrow \pi \Sigma}$, respectively. In Figs. 2 and 3 we plot the modulus square of these two quantities multiplied by the $\pi \Sigma$ momentum as a function of the energy. We also show the contribution of each resonance by itself (dotted and dashed lines). In both cases one only sees one resonant shape (solid line) but the simulated $T_{\pi \Sigma \rightarrow \pi \Sigma}$ amplitude in Fig. [3 produces a resonance at a lower energy and with a larger width. This case reproduces very well the nominal experimental $\Lambda(1405)$, both in the position and width. Actually, what is done in Ref. [5] to get the shape of the $\Lambda(1405)$ is precisely to plot the invariant mass distribution of the $\pi \Sigma$ states according to the expression

$$
\frac{d \sigma}{d M_{i}}=C\left|T_{\pi \Sigma \rightarrow \pi \Sigma}\right|^{2} q_{\text {c.m. }},
$$

where $C$ is a constant. However, if the invariant mass distribution of the $\pi \Sigma$ states were dominated by the $\bar{K} N \rightarrow \pi \Sigma$ amplitude, then the second resonance $R_{2}$ would be weighted more, since it has a stronger coupling to the $\bar{K} N$ state, resulting into an apparent narrower resonance peaking at higher energies as illustrated in Fig. 22 In order to avoid such ambiguities, in the calculation of the $\pi \Sigma$ invariant mass distribution of Ref. [3] a coupled channel scheme was implemented from the onset, not only to obtain the strong $\mathrm{S}$-wave $T$-matrix, but also in the production mechanism of the $I=0 \pi \Sigma$ state, involving initial $\pi \Sigma$ and $\bar{K} N$ states. The weight of the $K^{-} p$ channel relative to the $\pi^{-} \Sigma^{+}$one in the production mechanism, obtained in Ref. [3] from a fit to the data, is $r_{K^{-}} / r_{\pi^{-} \Sigma^{+}}=1.416$, being the same for any other $\bar{K} N$ or $\pi \Sigma$ channel, since the source has isospin $I=0$. The usual Eq. (28) is obtained from this formalism when $r_{\bar{K} N}=0$. However, in Ref. [3] a good description of the data is achieved when the $\bar{K} N$ channel is allowed to participate directly in the production mechanism as well $\left(r_{\bar{K} N} \neq 0\right)$ so that finally only one effective resonant shape is visible, despite of being determined by two rather narrow poles (see Table 3). Let us note that in Refs. [5, 10, the first pole in Table 1 has a width twice as large as that of the corresponding pole of Ref. [3], giving rise to a good reproduction of the $\pi \Sigma$ invariant mass distribution in terms of only the $T_{\pi \Sigma \rightarrow \pi \Sigma}$ amplitude, the one appearing in Eq. (28). This is no longer possible in Ref. [3] because the first pole is much narrower and the aforementioned coupled channel formalism is unavoidable in order to find agreement with data. For further details related to the formalism see Refs. [3, 24]. This discussion makes clear that a theoretical investigation of the rates $r_{\bar{K} N}$ and $r_{\pi \Sigma}$ from different reactions is a key ingredient for a complete understanding of the dynamics of the $\Lambda(1405)$ state.

We now turn to more realistic calculations in which we use the amplitudes generated in the chiral unitary approach of Ref. [10], rather than the approximated ones of Eqs. (26) and (27). In Fig. 4, we show the $\pi \Sigma$ invariant mass distributions constructed from the 


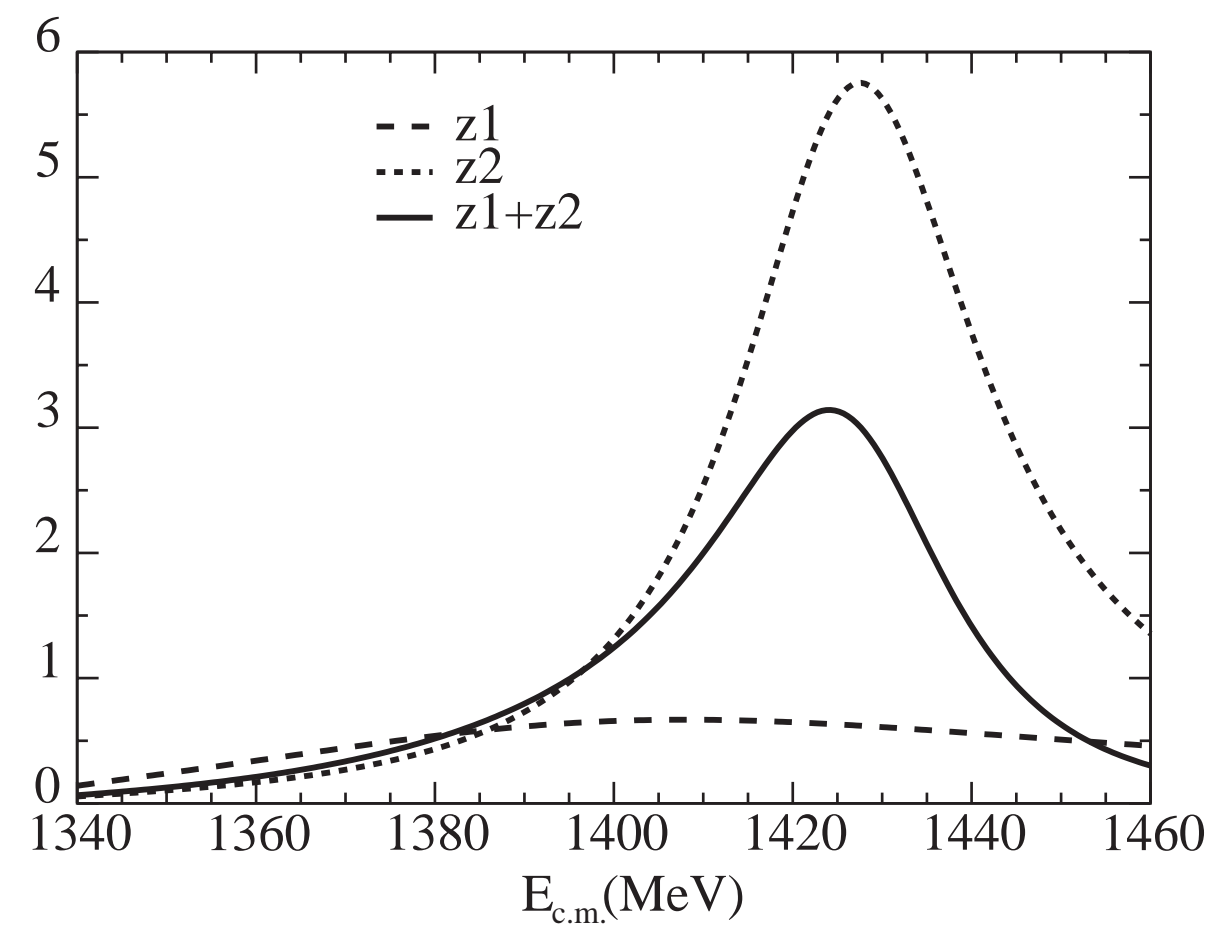

Figure 2: $z_{1}$ : the modulus square of the first term in Eq. (26) multiplied by $p_{\pi}$. $z_{2}$ : Same for the second term in Eq. (26) $z_{1}+z_{2}$ : Same for the coherent sum of the two terms.

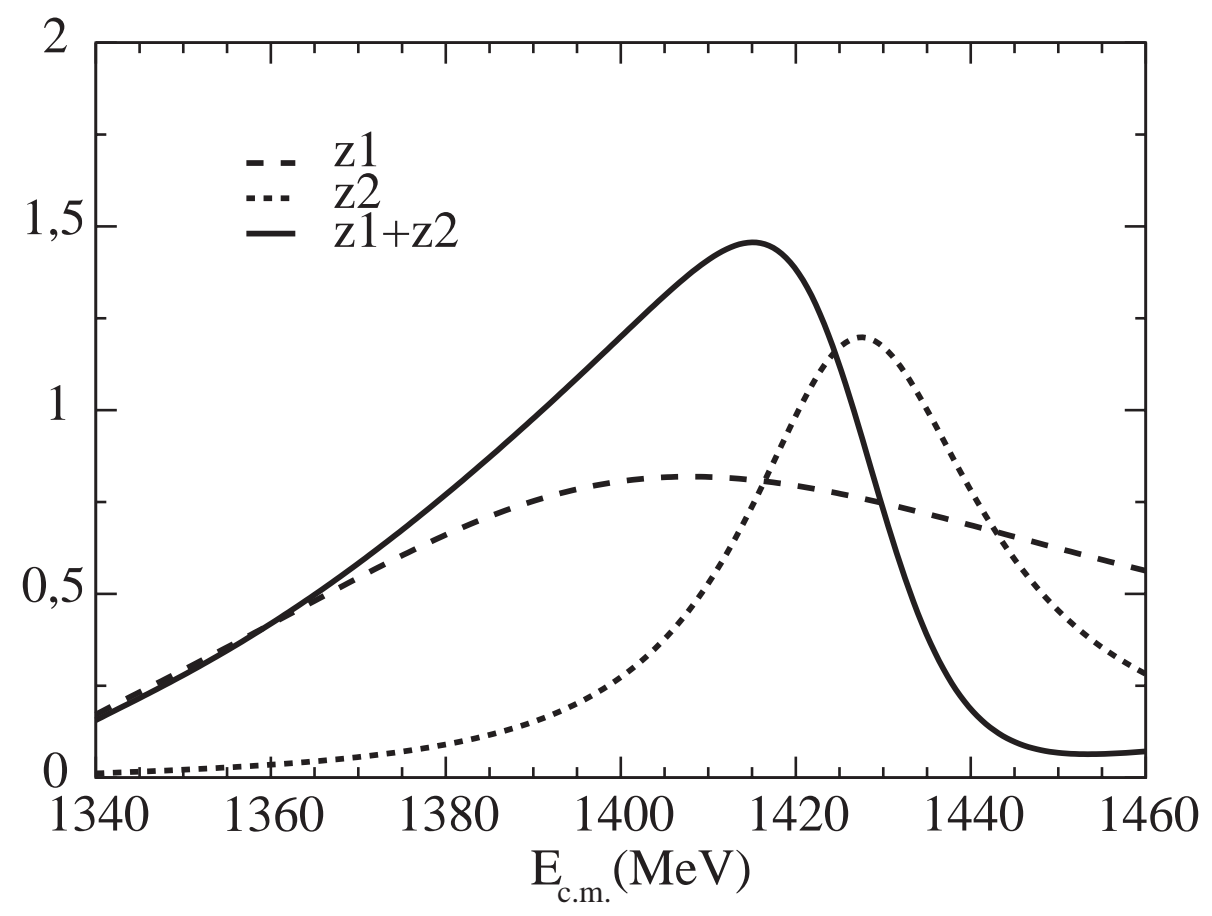

Figure 3: Same as Fig. 2 but for the terms in Eq. (27). 


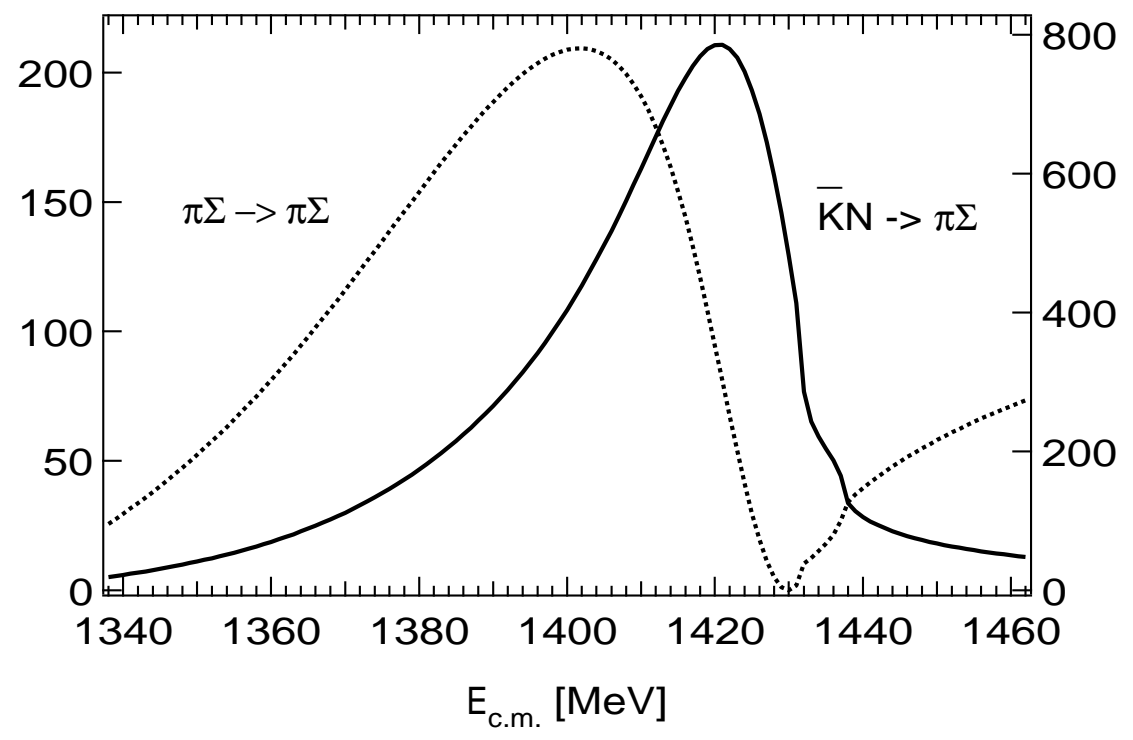

Figure 4: The $\pi \Sigma$ mass distributions with $I=0$ constructed from the $\bar{K} N \rightarrow \pi \Sigma$ and $\pi \Sigma \rightarrow \pi \Sigma$ amplitudes. The solid and dashed lines denote $\left|T_{\bar{K} N \rightarrow \pi \Sigma}\right|^{2} q_{\pi}$ and $\left|T_{\pi \Sigma \rightarrow \pi \Sigma}\right|^{2} q_{\pi}$, respectively. Units are arbitrary.

$\pi \Sigma \rightarrow \pi \Sigma$ (dotted line) and $\bar{K} N \rightarrow \pi \Sigma$ (solid line) amplitudes. As we expected, in the $\bar{K} N \rightarrow \pi \Sigma$ case, the resonance peaks at a higher energy and shows a narrower width. In Fig. [5 we include the corresponding loop function $G_{l}$ and plot the quantities $\left|G_{\pi \Sigma} T_{\pi \Sigma \rightarrow \pi \Sigma}\right|^{2}$ (dotted line) and $\left|G_{\bar{K} N} T_{\bar{K} N \rightarrow \pi \Sigma}\right|^{2}$ (solid line), which would appear in production processes of the $\Lambda(1405)$ assuming the build-up of the resonance in the multiple scattering is initiated by a $\pi \Sigma$ or $\bar{K} N$ state, respectively. It is interesting to see that in the case of the $\bar{K} N$ initial state the peak is narrower and appears at higher energy. This is another important result of this paper.

We can see that the results of the full calculation are very similar to those obtained with the qualitative model which allowed us to see that, in spite of starting from two quite different resonance poles, the result in an experiment would be to see a single resonant form. Yet, the shape and width could be different in different processes, depending on the weight that is given to the physical channels that initiate the resonance.

It is clear that, should there be a reaction which forces the initial channels to be $\bar{K} N$, then this would give more weight to the second resonance, $R_{2}$, and hence produce a distribution with a shape corresponding to an effective resonance narrower than the nominal one and at higher energy. Such a case indeed occurs in the reaction $K^{-} p \rightarrow$ $\Lambda(1405) \gamma$ studied theoretically in Ref. 25]. It was shown there that since the $K^{-} p$ system has a larger energy than the resonance, one has to lose energy emitting a photon prior to the creation of the resonance and this is effectively done by the Bremsstrahlung from the original $K^{-}$or the proton. Hence the resonance is initiated from the $K^{-} p$ channel. 


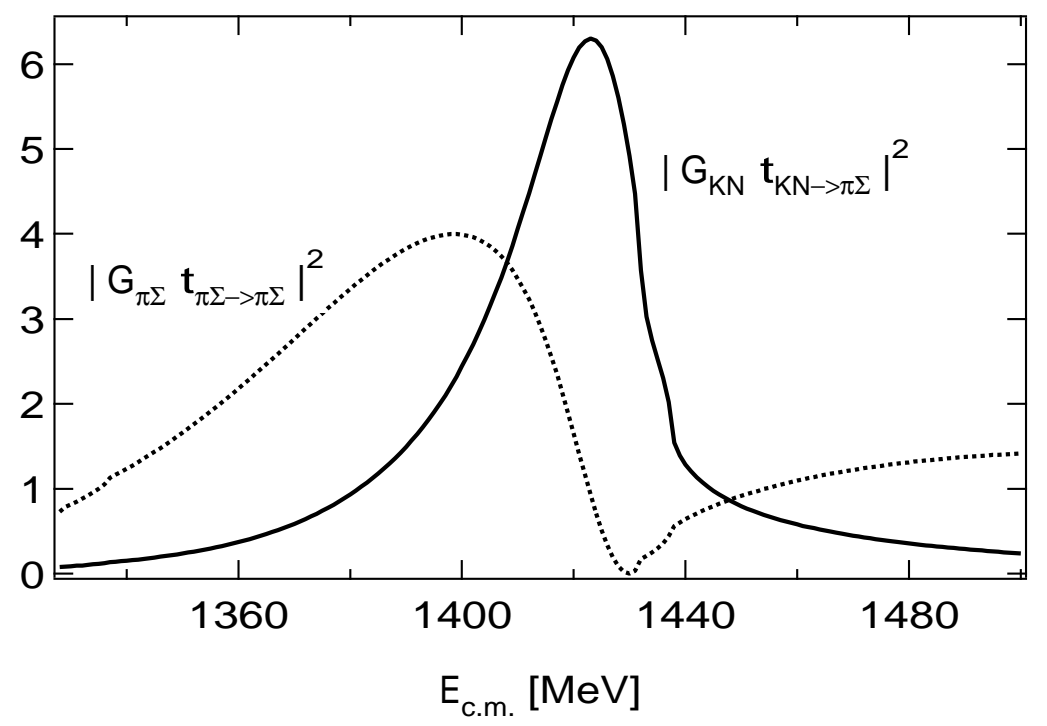

Figure 5: Modulus squared of the loop function $G_{l}$ times the amplitudes, simulating a reaction with the resonance initiated by the $\pi \Sigma$ (dashed line) or the $\bar{K} N$ (solid lines) channels.

\section{Conclusions}

In this paper we have investigated the poles appearing in the meson-baryon scattering matrix for strangeness $S=-1$ within a coupled-channel chiral unitary approach, using two different methods for breaking the SU(3) symmetry which have been used in the literature.

In both approaches some resonances are generated dynamically from the interaction of the octet of pseudoscalar mesons with the octet of the $1 / 2^{+}$baryons. The underlying $\mathrm{SU}(3)$ structure of the Lagrangians implies that, from the combination of the two original octets, a singlet and two octets of dynamically generated resonances should appear, but the dynamics of the problem makes the two octets degenerate in the case of exact $\mathrm{SU}(3)$ symmetry. The same chiral Lagrangians have mechanisms for chiral symmetry breaking which have as a consequence that the degeneracy is broken and two distinct octets appear. In Ref. [3] the interaction kernel $V$ is calculated strictly at lowest order in the chiral expansion (with a common baryon mass $M_{0}$ ), including consistently at this order the direct and crossed one baryon exchange contributions, while Refs. [5, 10] neglect the latter and utilize the physical baryon masses. In addition in ref. 3] a unique substraction constant is employed, as corresponds to the $\mathrm{SU}(3)$ limit, see appendix A, while in Refs. [5, 10] the subtraction constants are different from channel to channel and parameterized in terms of a cut-off. As a consequence the $\mathrm{SU}(3)$ breaking as well as the interacting kernel $V$ itself are different between Refs. [5, 10] and Ref. [3]. Although the SU(3) symmetry breaking mechanisms can be more general, and can be systematically included by the evaluation of 
$V$ to increasingly higher orders in a chiral expansion as shown in ref. [3], the two approaches followed in the present study, despite their differences, have shown very good agreement with the experimental observables in different $\bar{K} N$ reactions, thus giving support to our conclusions.

The breaking of the octet degeneracy has a a consequence that, in the physical limit, one of the $I=0$ octet poles appears quite close to the singlet pole, and both of them are very close to the nominal $\Lambda(1405)$. These two resonances are quite close but different, the one at lower energies with a larger width and a stronger coupling to the $\pi \Sigma$ states than the one at higher energies, which couples mostly to the $\bar{K} N$ states. This is the main finding of the present work, thus we conclude that there is not just one single $\Lambda(1405)$ resonance, but two, and that what one sees in experiments is a superposition of these two states.

Another interesting finding of the paper is the suggestion that it is possible to find out the existence of the two resonances by performing different experiments, since in different experiments the weights by which the two resonances are excited are different. In this respect we call the attention to one reaction, $K^{-} p \rightarrow \Lambda(1405) \gamma$, which gives much weight to the resonance which couples strongly to the $\bar{K} N$ states and, hence, leads to a peak structure in the invariant mass distributions which is narrower and appears at higher energies than the experimental $\Lambda(1405)$ peaks observed in hadronic experiments performed so far.

The two different approaches discussed in the paper to break $\mathrm{SU}(3)$ symmetry have also served to give an idea about the uncertainties of our predictions, resulting from higher order chiral terms in the calculation of interaction kernel $V$ not considered here. The relatively good agreement of the two approaches gives us confidence about the conclusions of the paper concerning the existence of the two $\Lambda(1405)$ resonances and their different coupling to the meson-baryon states. This important theoretical finding should stimulate new experiments exciting the $\Lambda(1405)$ resonance, as well as new analyses to unravel the double pole structure of the peaks seen in the reactions.

\section{Acknowledgments}

One of us, E.O., would like to acknowledge useful discussions with B. Holstein and J. Gasser on issues of this paper. We would also like to acknowledge the encouragement of $\mathrm{H}$. Toki for us to make this work and his collaboration in former works which stimulated us to do the present one. This work is partially supported by DGICYT projects BFM20001326, BFM2001-01868, FPA2002-03265, the EU network EURIDICE contract HPRN-CT2002-00311, and the Generalitat de Catalunya project 2001SGR00064. D.J. would like to acknowledge the support of Japanese Ministry of Education, Culture, Sports, Science and Technology to stay at IFIC, University of Valencia, where part of this work was done. 


\section{A Subtraction constants in the $\mathrm{SU}(3)$ limit}

In this appendix we want to show how the $a_{i}$ subtraction constants, present in the $G_{i}(s)$ functions, become equal in the $\mathrm{SU}(3)$ limit, which is assumed to hold in all this appendix.

Each SU(3) irreducible representation decouples and then, analogously to Eq. (11) which is given for the physical channels, we will have for each irreducible representation $\gamma$ the amplitude:

$$
T_{\gamma}=\frac{V_{\gamma}}{1-V_{\gamma} G_{\gamma}}
$$

where now all the functions in the previous formula are just numbers due to the decoupling of different irreducible representations. The subtraction constant of $G_{\gamma}$ is denoted by $a_{\gamma}$.

Nevertheless, we can still use Eq. (11) and deduce the $T_{\gamma}$ amplitudes by an orthonormal transformation. Correspondingly, we are taking the SU(3) Clebsch-Gordan coefficients real so that they satisfy:

$$
\begin{aligned}
& \sum_{i}\langle i, \gamma\rangle\langle i, \mu\rangle=\delta_{\gamma \mu} \\
& \sum_{\gamma}\langle i, \gamma\rangle\langle j, \gamma\rangle=\delta_{i j}
\end{aligned}
$$

where the latin indexes refer to the physical channels and the greek ones to the $\mathrm{SU}(3)$ eigenstates. Eq. (11), which applies to the physical channels, can be rewritten as:

$$
T_{i j}=V_{i j}+\sum_{k, k^{\prime}} V_{i k} G_{k k^{\prime}} T_{k^{\prime} j}
$$

where $G_{k k^{\prime}}$ is a diagonal matrix, $G_{k k^{\prime}}=G_{k} \delta_{k k^{\prime}}$, as discussed in section 2, Hence, by making a change of basis, we find:

$$
\sum_{i j}\langle i, \gamma\rangle A_{i j}\langle j, \mu\rangle=A_{\gamma \mu}=A_{\gamma} \delta_{\gamma \mu},
$$

with $A$ standing for $V, G$ or $T$, and we have used the fact that in the $\operatorname{SU}(3)$ limit $V, G$ and $T$ are singlet operators. Inverting the previous equation for $A \equiv G$ we find:

$$
G_{k k^{\prime}}=\sum_{\mu}\langle k, \mu\rangle G_{\mu}\left\langle k^{\prime}, \mu\right\rangle,
$$

and thus,

$$
\begin{aligned}
\sum_{k^{\prime}} G_{k k^{\prime}}\left\langle k^{\prime}, \gamma\right\rangle & =\sum_{k^{\prime}} \sum_{\mu}\langle k, \mu\rangle G_{\mu}\left\langle k^{\prime}, \mu\right\rangle\left\langle k^{\prime}, \gamma\right\rangle \\
& =\langle k, \gamma\rangle G_{\gamma} .
\end{aligned}
$$

Using now that $G_{k k^{\prime}}$ is diagonal, one has,

$$
G_{k}\langle k, \gamma\rangle=G_{\gamma}\langle k, \gamma\rangle .
$$


Since this relation is valid for all $k$ and all $\gamma$, it is sufficient to take several physical states which have components in different $\mathrm{SU}(3)$ representations ${ }^{1}$, to see that all the $G_{\gamma}$ functions must be the same, and as a consequence, all the $G_{k}$ are also equal. Equivalently, the subtraction constants $a_{\gamma}$ turn out to be the same in the $\mathrm{SU}(3)$ limit and, consequently, the subtraction constants $a_{k}$ are independent of the physical channel.

\section{B Deviations of the pole positions from the $\mathrm{SU}(3)$ limit}

In this appendix we would like to show the direction of deviation of the pole positions from the $\mathrm{SU}(3)$ symmetric limit when an infinitesimal $\mathrm{SU}(3)$ breaking is assumed. In the $\mathrm{SU}(3)$ symmetric limit, we find two bound states, one corresponds to the singlet and the other corresponds to the degenerate octets $\left(8_{s}\right.$ and $\left.8_{a}\right)$. Due to the $\mathrm{SU}(3)$ breaking effects, the isospin $I=0,1$ states of the two octets $\left(8_{s}\right.$ and $\left.8_{a}\right)$ split apart in four states as shown in Fig. 1.

The energies of the bound states are calculated as the solutions $z$ of the secular equation:

$$
\operatorname{det}[1-V(z) G(z)]=0 .
$$

In the $\mathrm{SU}(3)$ symmetric limit, the scattering amplitude is expressed as a diagonal matrix in the basis of the $\mathrm{SU}(3)$ irreducible representations, as given in Eq. (A.1). For the diagonal matrix the determinant is written in the product from:

$$
\prod_{\gamma}\left(1-V_{\gamma}\left(z_{0}^{\gamma}\right) G_{0}\left(z_{0}^{\gamma}\right)\right)=0
$$

where $z_{0}^{\gamma}$ denotes the solution for the irreducible representation $\gamma$ in the $\mathrm{SU}(3)$ symmetric limit and the loop integral $G_{0}$ is independent of the irreducible representation in this limit, as discussed in appendix $\mathrm{A}$

Now let us introduce an infinitesimal breaking of the SU(3) symmetry to the $G$ function through the subtraction constants and the masses of baryons and mesons as in Eq. (10). We expand the $G$ function in term of the $\mathrm{SU}(3)$ breaking parameter $x$ :

$$
G_{\gamma}=G_{0}+\tilde{G}_{\gamma} \delta x
$$

where $\delta x \ll 1$ and

$$
\tilde{G}_{\gamma}=\left.\frac{\partial G_{\gamma}}{\partial x}\right|_{x=0}=\left.\sum_{i}\langle i, \gamma\rangle \frac{\partial G_{i}}{\partial x}\right|_{x=0}\langle i, \gamma\rangle .
$$

In general, the $G$ function in the $\mathrm{SU}(3)$ basis has off-diagonal components when the $\mathrm{SU}(3)$ breaking effects are introduced. Nevertheless, for small $\delta x$, such off-diagonal components

\footnotetext{
${ }^{1}$ Let us note that since $G$ is a $\mathrm{SU}(3)$ singlet operator, its matrix elements in the $\mathrm{SU}(3)$ basis are $G_{\gamma}$ times the identity matrix for the states belonging to the same irreducible representation.
} 
contribute to higher orders when the energies of the bound states are calculated using Eq. (B.1). Therefore, the equation determining the positions of the poles is obtained again in the decoupled form:

$$
\prod_{\gamma}\left(1-V_{\gamma}\left(z^{\gamma}\right)\left\{G_{0}\left(z^{\gamma}\right)+\tilde{G}\left(z^{\gamma}\right) \delta x\right\}\right)=0 .
$$

Regarding $\tilde{G} \delta x$ as a perturbation, we can calculate deviations of the positions of the poles due to a small $\mathrm{SU}(3)$ breaking effect. Writing $z^{\gamma}=z_{0}^{\gamma}+\epsilon_{\gamma}$, we obtain

$$
\epsilon_{\gamma}=-\left.\frac{V_{\gamma} \tilde{G}_{\gamma}}{V_{\gamma}^{\prime} G_{0}+G_{0}^{\prime} V_{\gamma}} \delta x\right|_{z=z_{0}^{\gamma}},
$$

where $V^{\prime}$ and $G_{0}^{\prime}$ are derivatives of the $V$ and $G$ functions with respect to the energy $z$. In the case of the octets, the sign of $\epsilon_{\gamma}$ depends only on $\tilde{G}_{\gamma}$. Here we show the numerical results of the deviations at $\delta x=0.05$ :

$$
\begin{array}{cll}
\epsilon_{1}=1.2[\mathrm{MeV}] & \epsilon_{8_{s}, I=0}=-0.30[\mathrm{MeV}] & \epsilon_{8_{a}, I=0}=5.7[\mathrm{MeV}] \\
& \epsilon_{8_{s}, I=1}=4.1[\mathrm{MeV}] & \epsilon_{8_{a}, I=1}=-1.6[\mathrm{MeV}]
\end{array},
$$

which explain the deviations in the positions of the poles with increasing $x$ observed in Fig. [1.

\section{References}

[1] M. Jones, R. H. Dalitz and R. R. Horgan, Nucl. Phys. B 129 (1977) 45.

[2] N. Kaiser, T. Waas and W. Weise, Nucl. Phys. A 612 (1997) 297.

[3] J. A. Oller and U.-G. Meißner, Phys. Lett. B 500 (2001) 263.

[4] U.-G. Meißner, J. A. Oller, Phys. Rev. D 64 (2001) 014006.

[5] E. Oset and A. Ramos, Nucl. Phys. A 635 (1998) 99.

[6] P. J. Fink, G. He, R. H. Landau and J. W. Schnick, Phys. Rev. C 41 (1990) 2720.

[7] D. Jido, A. Hosaka, J. C. Nacher, E. Oset and A. Ramos, Phys. Rev. C 66 (2002) 025203.

[8] C. Garcia-Recio, J. Nieves, E. Ruiz Arriola and M. J. Vicente Vacas, arXiv:hep-ph/0210311.

[9] G. Ecker, Prog. Part. Nucl. Phys. 35 (1995) 1; V. Bernard, N. Kaiser and U.G. Meißner, Int. J. Mod. Phys. E 4 (1995) 193; A. Pich, Rept. Prog. Phys. 58 (1995) 563.

[10] E. Oset, A. Ramos and C. Bennhold, Phys. Lett. B 527 (2002) 99 [Erratum-ibid. B 530 (2002) 260].

[11] J. A. Oller and E. Oset, Nucl. Phys. A 620 (1997) 438 [Erratum-ibid. A 652 (1999) 407]. 
[12] J. L. Goity, C. L. Schat and N. N. Scoccola, Phys. Rev. D 66 (2002) 114014; Phys. Rev. Lett. 88 (2002) 102002.

[13] J. A. Oller and E. Oset, Phys. Rev. D 60 (1999) 074023.

[14] A. Ramos, E. Oset and C. Bennhold, Phys. Rev. Lett. 89 (2002) 252001.

[15] T. Inoue, E. Oset and M. J. Vicente Vacas, Phys. Rev. C 65 (2002) 035204.

[16] N. Kaiser, P. B. Siegel and W. Weise, Nucl. Phys. A 594 (1995) 325.

[17] N. Kaiser, Eur. Phys. J. A 3 (1998) 307.

[18] J. A. Oller, E. Oset and J. R. Peláez, Phys. Rev. D 59 (1999) 074001 [Erratum-ibid. D 60 (1999) 099906].

[19] T. Hyodo, S. I. Nam, D. Jido and A. Hosaka, arXiv:nucl-th/0212026.

[20] J. C. Nacher, E. Oset, H. Toki and A. Ramos, Phys. Lett. B 455, 55 (1999).

[21] J. K. Ahn et al., Proceedigns of the XVI Particles and Nuclei International Conference (PANIC02), Eds. K. Imai, T. Kishimodo and H. Toki, Nucl. Phys. A, in print.

[22] H. Georgi, Lie Algebras in Particle Physics, Frontiers in Physics.

[23] D. B. Lichtenberg, Unitary Symmetry and Elementary Particles, Academic Press.

[24] U.-G. Meißner and J. A. Oller, Nucl. Phys. A 679 (2001) 671.

[25] J. C. Nacher, E. Oset, H. Toki and A. Ramos, Phys. Lett. B 461 (1999) 299. 